\title{
Histological heterogeneity of glomerular segmental lesions in focal segmental glomerulosclerosis
}

\author{
Sekiko Taneda $\cdot$ Kazuho Honda $\cdot$ Keiko Uchida $\cdot$ \\ Kosaku Nitta $\cdot$ Wako Yumura $\cdot$ Hideaki Oda • \\ Michio Nagata
}

Received: 27 October 2010/Accepted: 28 February 2011/Published online: 19 March 2011

(C) The Author(s) 2011. This article is published with open access at Springerlink.com

\begin{abstract}
Focal segmental glomerulosclerosis (FSGS) involves considerable histological heterogeneity in terms of location and quality of the glomerular segmental lesions. The present study investigated the heterogeneity of segmental lesions in each variant of FSGS, determined by the Columbia classification, and its clinical relevance. All glomerular segmental lesions of 80 cases of primary FSGS were evaluated histologically based on location [tip (TIP), perihilar (PH), or not otherwise specified (NOS)], and quality (cellular or
\end{abstract}

Electronic supplementary material The online version of this article (doi:10.1007/s11255-011-9932-y) contains supplementary material, which is available to authorized users.

S. Taneda $\cdot$ K. Honda $(\bowtie) \cdot$ H. Oda

Department of Pathology, Tokyo Women's Medical University, 8-1 Kawada-cho, 162-8666 Shinjuku-ku, Tokyo, Japan

e-mail: honda@research.twmu.ac.jp

K. Uchida $\cdot$ K. Nitta

Department of Medicine, Kidney Center, Tokyo Women's Medical University, Tokyo, Japan

W. Yumura

Department of Nephrology, Jichi Medical University,

Tochigi, Japan

M. Nagata

Department of Kidney and Vascular Pathology, Graduates School of Comprehensive Human Sciences, University of Tsukuba, Ibaraki, Japan fibrous). Among the 1,299 glomeruli of the 80 biopsy specimens, 210 glomeruli $(16.2 \%)$ had segmental lesions, comprising 57 (27\%) cellular TIP, 4 (2\%) fibrous TIP, $42(20 \%)$ cellular NOS, 86 (41\%) fibrous NOS, and 21 (10\%) fibrous PH lesions. Each case was also classified into one of the five histological variants of the Columbia classification: collapsing (COL), TIP, cellular (CEL), PH, or NOS. Overlap of segmental lesions in different location categories was seen in the COL, TIP, and PH variants, and heterogeneity of quality was apparent in the COL and CEL variants. Histological findings of the CEL variant (endocapillary hypercellularity) were observed in nine of the $13 \mathrm{COL}$ variants. Both location and quality correlated with disease duration, degree of proteinuria, and histological severity of global glomerular sclerosis and tubulointerstitial lesions. These results demonstrated the histological heterogeneity of glomerular segmental lesions in all variants of the Columbia classification, except NOS. However, the fidelity of location and dominance of histological features were generally conserved in the TIP and PH variants. The COL and CEL variants warrant further investigation because of their overlapping histological findings and apparent histological heterogeneity in the glomerular segmental lesions.

Keywords Focal segmental glomerulosclerosis · Columbia classification - Segmental lesion .

Perihilar · Tip · Collapsing · Not otherwise specified · Cellular · Fibrous 


\section{Introduction}

Focal segmental glomerulosclerosis (FSGS) is a morphologically defined renal disease characterized by diverse clinical manifestations. Many patients with FSGS display a progression to advanced glomerular sclerosis and subsequent end-stage renal disease (ESRD) [1]. The etiology of FSGS is typically unknown but recent investigations have revealed that many distinct factors, including a genetic abnormality, are involved in the pathogenesis of secondary FSGS, affecting the disturbance of podocytes and slit diaphragm morphology and function [2].

Recently, a working formulation for the morphological classification of primary FSGS, the "Columbia classification," was proposed by D'Agati et al. [3]. It includes five histological variants: collapsing (COL), tip (TIP), cellular (CEL), perihilar (PH), and not otherwise specified (NOS). Several studies have reported significant differences in the clinical features and outcomes among patients with different histological variants of FSGS, based on the Columbia classification [4-8]. The utility of the Columbia classification in renal transplant allografts has been studied recently $[9,10]$. In one study, the majority of recurrent FSGS presented the same variant as the original FSGS in the native kidney [9], whereas another study found that the variant of FSGS in the native kidneys was not predictive of either recurrence or the type of FSGS observed in the allograft [10]. Thus, it remains unclear whether different Columbia variants represent different pathophysiological entities of FSGS or different stages of the same pathophysiological entity.

A detailed histological observation of glomerular segmental lesions in FSGS indicated that patients with severe clinical manifestation often presented similar histological features, such as proliferation of intrinsic glomerular cells comprising epithelial and endothelial cells. The accumulation of infiltrating leukocytes and foam cells within the glomerular capillary was also observed in the segmental lesions. Conversely, patients with advanced glomerular sclerosis and impaired renal function generally exhibited a consolidation of glomerular tufts with an increase of extracellular matrix, resulting in segmental glomerular sclerosis [11]. These histological qualities of the segmental lesions are reflected by a mixture of activity and chronicity of the glomerular inflammatory process. The histological activity and chronicity significantly correlate with the clinical manifestations and outcomes in various glomerular diseases. A recently revised classification for lupus nephritis [12] and similar attempts for IgA nephropathy [13-17] that consider the histological activity and chronicity of glomerular lesions appear to be beneficial for the clinical management of kidney diseases.

In the Columbia classification, the variants COL, TIP, and CEL predominantly display different aspects of histological activity, such as glomerular epithelial cell proliferation and intracapillary hypercellularity, whereas the variants $\mathrm{PH}$ and NOS usually show histological chronicity, such as consolidation of glomerular tufts. However, it is uncertain whether variants contain a uniform or mixed histological quality (activity/chronicity) of segmental lesions, and whether the histological quality has implications for clinical manifestation. Furthermore, it is not known whether a mutual relationship exists between the location and quality of glomerular segmental lesions.

In the present study, we evaluated histologically the glomerular segmental lesions from 80 cases of primary FSGS with respect to location and histological quality. Each case was further classified into one of the five histological variants of the Columbia FSGS classification, and the histological heterogeneity of each variant was analyzed. Our findings indicate the presence of histological heterogeneity in each variant of the Columbia classification and the importance of quality and location for assessing disease activity in patients with FSGS.

\section{Materials and methods}

Eighty biopsy-proven cases of primary FSGS seen at the Department of Medicine, Kidney Center, Tokyo Women's Medical University between 1982 and 2007 were enrolled in the study. Five glomeruli per biopsy specimen was the minimum requirement for enrollment in this study. The average number of glomeruli per one biopsy specimen was 16.2 \pm 11.7. FSGS was diagnosed based on the presence of glomerular segmental lesions in at least one glomerulus in 40 consecutive sections. A segmental lesion was defined as a consolidation of the glomerular tuft due to luminal collapse and a subsequent increase of cellular 
components and extracellular matrix. Hyalinosis was occasionally associated with the segmental lesions. The cellular components consisted of intrinsic glomerular cells, such as glomerular epithelial, endothelial and mesangial cells, and infiltrating leukocytes and foam cells (macrophages).

The diagnostic algorithm of FSGS is described in Fig. 1. Patients diagnosed with other primary glomerular diseases, collagen diseases, systemic vasculitis, or diabetes were excluded. No patient was diagnosed with malignant hypertension, morbid obesity, viral infection, or drug-induced nephropathy. The subjects in this study were all adults (range, 18-77 years-old, average age: $39.4 \pm 1.7$ ). There was no familial clustering of FSGS in these patients. Thus, the subjects were diagnosed mostly with primary (idiopathic)

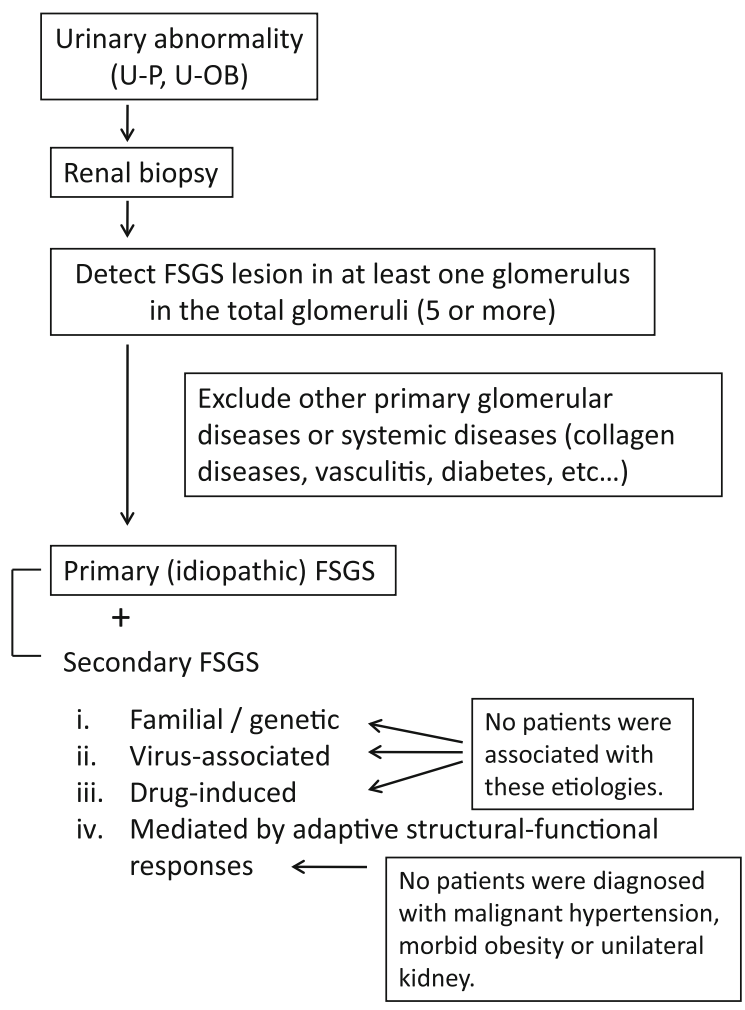

Fig. 1 Screening program for diagnosis and etiologies of FSGS. FSGS was diagnosed based on the presence of glomerular segmental lesions in at least one glomerulus. Patients diagnosed with other primary glomerular diseases or systemic disease, such as collagen diseases, vasculitis, or diabetes, were excluded. No patient was identified with a cause for secondary FSGS. Thus, the subjects of this study were diagnosed mostly with primary (idiopathic) FSGS
FSGS, but the possibility of secondary FSGS, mediated by adaptive glomerular changes to hypertension or non-morbid obesity, could not be completely excluded.

All subjects agreed to participate in this study and provided written informed consent.

Clinical data of the 80 patients with primary FSGS

Renal function was evaluated based on the serum creatinine levels $(\mathrm{sCr}, \mathrm{mg} / \mathrm{dl})$ at the time of biopsy and the end of follow-up. The level of proteinuria (g/day) was also evaluated at biopsy and final observation. The interval (months) between the discovery of proteinuria and the kidney biopsy was regarded as the disease duration. Nephrotic-range proteinuria was defined as urinary protein excretion $(\mathrm{U}-\mathrm{p}): \geq 3.0 \mathrm{~g} /$ day. Hypertension was defined as systolic blood pressure of $\geq 150 \mathrm{mmHg}$ and diastolic blood pressure of $\geq$ $90 \mathrm{mmHg}$. Malignant hypertension was defined as diastolic blood pressure of $\geq 130 \mathrm{mmHg}$ accompanied by papilledema or retinal hemorrhage. Obesity was defined as a body mass index (BMI) $\geq 30 \mathrm{~kg} / \mathrm{m}^{2}$, and morbid obesity was defined as a BMI $\geq 40 \mathrm{~kg} / \mathrm{m}^{2}$. Remission of FSGS was defined as a reduction of proteinuria to less than $0.5 \mathrm{~g} /$ day. End-stage renal disease (ESRD) was defined as a clinical state requiring regular renal replacement therapy.

Variant classification and histological evaluation of glomerular segmental lesions in FSGS

Three pathologists (ST, KH, and $\mathrm{MN}$ ) jointly reviewed the glomerular segmental lesions using a multi-head optical microscope to reach a consensus diagnosis for location and quality of lesions, and the variant of the Columbia classification. Glomerular segmental lesions were evaluated by their location and quality, according to the following procedures: The location of segmental lesion in each glomerulus was first categorized into three types: tip (TIP), perihilar (PH), or not otherwise specified (NOS). The TIP area was defined as the outer $25 \%$ of the glomerular tuft around the tubular pole, the $\mathrm{PH}$ area was defined as the proximal $25 \%$ of the glomerular tuft around the vascular pole, and any location that was not in the TIP or PH areas was considered to be NOS. Next, the quality of each segmental lesion was 
subtyped as cellular or fibrous, based on its predominant histological features. The cellular subtype was characterized by a proliferation of glomerular epithelial cells and/or intracapillary occlusion with hypercellularity, including foam cells and infiltrated leukocytes. The fibrous subtype was characterized by a consolidation of the glomerular tuft with increased extracellular fibrous matrix (sclerosis). Hyalinosis was observed in both the cellular and fibrous subtypes. Finally, each case was classified as one of the five histological variants of the Columbia classification: collapsing (COL), tip (TIP), cellular (CEL), perihilar (PH), or NOS [3].

Determination of dominant location and quality of segmental lesions

The dominant location and quality of the segmental lesions in all cases was determined using the following methods. The most frequent location pattern among all segmental lesions in a given case was assigned as the dominant location category of that case. When the number of lesions in each location category were the same, the priority order was defined as TIP $>\mathrm{PH}>$ NOS. The quality of the segmental lesions in each case was determined to be cellular when all lesions were cellular, fibrous when all lesions were fibrous, and fibrocellular when cellular and fibrous subtypes co-existed in the same biopsy specimen. Clinical and histological features were compared according to the dominant location category or quality subtype, respectively.

Grading of other histological lesions

The number of glomeruli exhibiting global sclerosis (GS) or segmental sclerosis (SS) was calculated as a percentage of the total number of glomeruli, GS or SS, respectively, in each specimen. Interstitial fibrosis and tubular atrophy (IF/TA) were graded semi-quantitatively, based on the affected cortical area, as a percentage of the total cortical area, where $0=$ absent or $<5 \%$, $1+=6-25 \%, 2+=26-50 \%$, and $3+=>50 \%$.

\section{Statistical analysis}

Continuous variables are presented as means \pm standard error (SEM). Statistical analysis between two groups was performed using Student's $t$-test or the
Mann-Whitney $U$-test, and analysis among multiple groups was performed using one-way or two-way ANOVA. Multiple comparisons (post hoc) using Scheffe's test were performed to compare continuous variables between two groups. The ratio of patients with ESRD was compared by Ryan's test. Analyses were performed using the SAS software (ver. 9.1.3; SAS Institute, Inc., Cary, NC, USA), and Ryan's test was performed using the ' $R$ ' statistical analysis package. Statistical significance was accepted for $P$-values $<0.05$.

\section{Results}

Histological variants according to the Columbia classification

The 80 cases of FSGS were classified according to the Columbia classification as follows: COL, 13 cases (16.2\%); TIP, 24 cases (30.0\%); CEL, 11 cases (13.8\%); PH, 13 cases (16.2\%); and NOS, 19 cases (23.8\%; Fig. 2). The correspondence between the variant obtained by Columbia classification and the category determined by the dominant location and quality factor is shown in Table 1 . The 13 cases of the COL variant included cases categorized as TIP cellular (2 cases), NOS cellular (4), NOS fibrocellular (5), and PH fibrocellular (2). Most of the 24 TIP variant cases were categorized as TIP cellular (18 cases), with three cases of TIP fibrocellular, one of

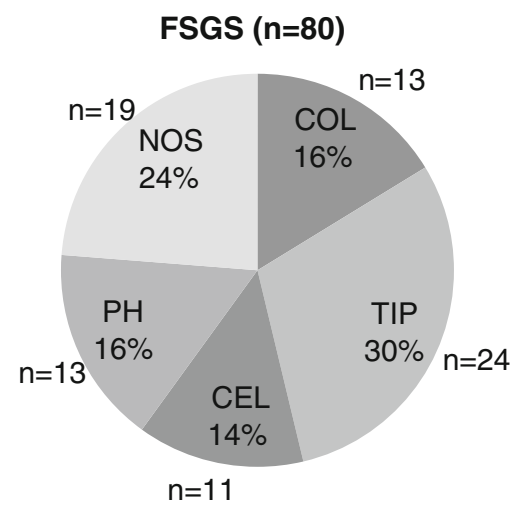

Fig. 2 Histological variants according to the Columbia classification. The 80 cases of FSGS were classified according to the Columbia classification as follows: COL, 13 cases (16.2\%), TIP, 24 cases $(30.0 \%)$, CEL, 11 cases (13.8\%), PH, 13 cases (16.2\%), and NOS, 19 cases $(23.8 \%)$ 
Table 1 Correspondence of the variant by Columbia classification to the category determined by dominant location/quality factor

\begin{tabular}{ll}
\hline $\begin{array}{l}\text { Columbia classification } \\
\text { (number of cases) }\end{array}$ & $\begin{array}{l}\text { Dominant location/quality } \\
\text { factor (number of cases) }\end{array}$ \\
\hline COL (13) & TIP-C (2) \\
& NOS-C (4) \\
& NOS-F/C (5) \\
& PH-F/C (2) \\
& TIP-C (18) \\
TIP (24) & TIP-F/C (3) \\
& NOS-F/C (1) \\
& NOS-F (2) \\
CEL (11) & NOS-C (5) \\
PH (13) & NOS-F/C (6) \\
NOS (19) & PH-F (13) \\
& NOS-F/C (1) \\
& NOS-F (18)
\end{tabular}

$C O L$ collapsing variant, TIP tip variant, $C E L$ cellular variant, $\mathrm{PH}$ perihilar variant, NOS not otherwise specified variant, $C$ cellular, $F / C$ fibrous/cellular, and $F$ fibrous

NOS fibrocellular, and two of NOS fibrous. Among the 11 cases of the CEL variant, there were five NOS cellular cases and six NOS fibrocellular cases. All 13 cases of the $\mathrm{PH}$ variant were categorized as $\mathrm{PH}$ fibrous. Nearly all of the 19 cases of the NOS variant were categorized as NOS fibrous (18), with one NOS fibrocellular case.

Baseline clinical findings and outcomes

A comparison of the baseline clinical findings among the FSGS variants is presented in Table 2. The histological variants showed significant differences in the mean interval between discovery of proteinuria and biopsy and in the mean levels of $\mathrm{sCr}$, serum albumin (sAlb), and urinary protein excretion at the time of the kidney biopsy (Table 2). The mean interval between discovery of proteinuria and biopsy was shortest for the TIP variant $(7.8 \pm 2.3$ months), followed by the CEL $(18.7 \pm 5.4$ months $)$ and COL variants (41.6 \pm 16.2 months), with considerably longer times for the NOS $(89.0 \pm 5.4$ months $)$ and $\mathrm{PH}$ variants $(136.0 \pm 27.7$ months $)$. The $\mathrm{sCr}$ level at biopsy was highest in the COL variant $(2.04 \pm$ $0.54 \mathrm{mg} / \mathrm{dl})$, followed by the CEL variant $(1.58 \pm$ $0.18 \mathrm{mg} / \mathrm{dl}$ ). The sAlb level at biopsy was lowest in the
CEL variant $(2.84 \pm 0.32 \mathrm{~g} / \mathrm{dl})$ and TIP variant $(2.88 \pm 0.20 \mathrm{~g} / \mathrm{dl})$, followed by the COL variant $(3.07 \pm 0.31 \mathrm{~g} / \mathrm{dl})$. Proteinuria at biopsy was high in the CEL $(8.9 \pm 2.3 \mathrm{~g} /$ day $)$, TIP $(8.8 \pm 2.3 \mathrm{~g} /$ day $)$, and $\mathrm{COL}$ variants $(7.1 \pm 2.6 \mathrm{~g} /$ day $)$, and was relatively low in the NOS $(1.7 \pm 0.5 \mathrm{~g} /$ day $)$ and $\mathrm{PH}$ variants $(2.1 \pm 0.5 \mathrm{~g} /$ day $)$. The prevalence of nephrotic syndrome was highest in TIP $(72.7 \%)$ and CEL $(72.7 \%)$ variants, followed by the COL variant (46.2\%), and much lower in PH (16.7\%) and NOS $(11.1 \%)$ variants.

The therapeutic modalities and outcomes among the variants are shown in Table 3. Steroids were usually administered, either alone or combined with immunosuppressive agents, in the COL (72.8\%), TIP (86.3\%), and CEL (70\%) variants. Angiotensin converting enzyme inhibitors (ACEI) or angiotensin II receptor blockers (ARB) were frequently administered to all variants $(41 / 75,52.4 \%)$, and ACEI/ARB administration without steroids was preferred in the $\mathrm{PH}$ variant (84.6\%). LDL apheresis was performed in two of the CEL cases and in one of the COL and TIP cases, to treat severe dyslipidemia. Sixty-six cases were followed and their outcomes were evaluated. Persistent proteinuria was evident in all variants; however, at final observation, the proteinuria levels were low and showed no difference among the variants. Additionally, the $\mathrm{sCr}$ level at the final evaluation did not differ among the variants, probably due to the small number of cases and the relatively short period of observation (55.9 \pm 8.8 months). The rate of remission was higher in the TIP $(82.2 \%)$ variant than in the other variants [CEL $(60 \%)$, NOS (47.1\%), COL (44.4\%), and PH (30\%)]. Twelve of the 66 cases (18.2\%) progressed to ESRD and began HD. These included two of the 19 cases with the TIP variant $(10.5 \%)$, and relatively higher percentages of those with $\mathrm{COL}(3 / 10,30 \%)$ and CEL variants $(3 / 9,33.3 \%)$, with no significant difference between the last two. No patient with $\mathrm{PH}$ progressed to ESRD.

\section{Histological findings}

Histological findings in a total of 1,299 glomeruli were assessed in the biopsy specimens of the 80 patients (Table 4). Among these, 207 glomeruli (15.9\%) were globally sclerotic, and 210 (16.2\%) were segmentally sclerotic, with an average of 


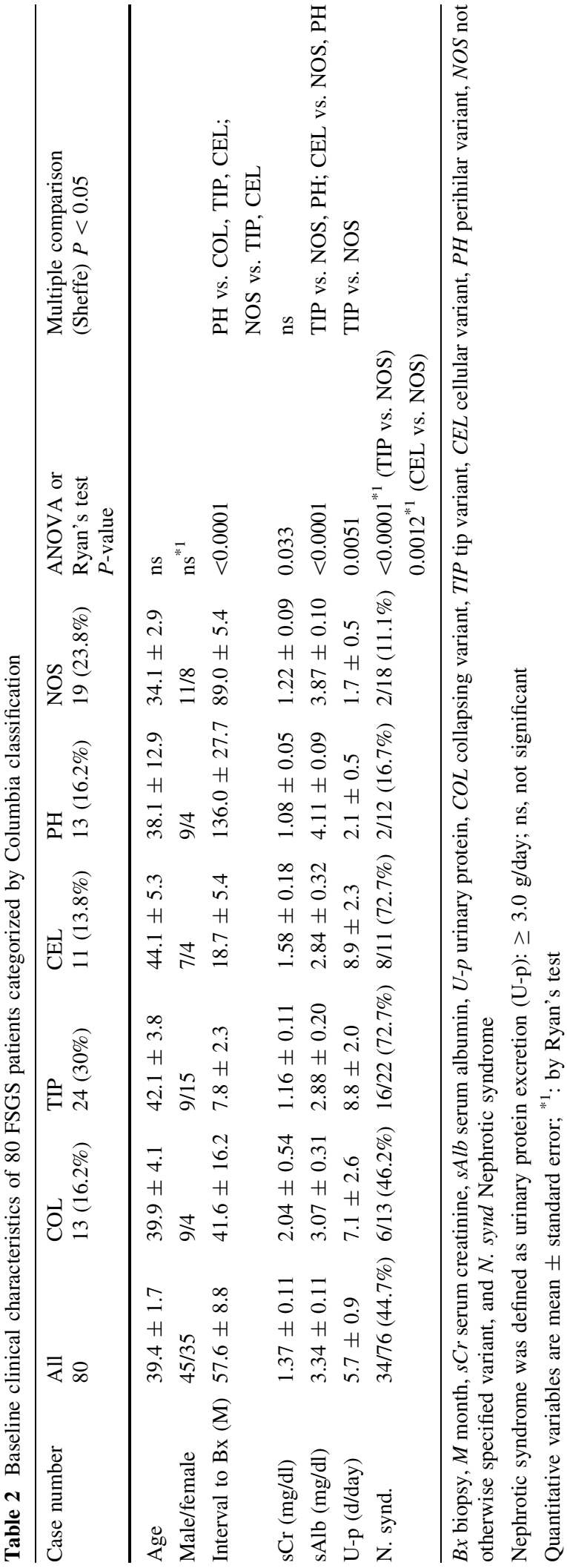

$2.63 \pm 0.20$ segmentally sclerotic glomeruli per biopsy specimen. The percentage of global glomerular sclerosis (\%GS) differed significantly among variants $(P=0.036)$, with higher values for the NOS $(23.6 \pm 5.1 \%)$ and COL variants $(21.1 \pm 5.9 \%)$, followed by the CEL $(17.5 \pm 4.1 \%)$ and PH variants $(16.8 \pm 2.9 \%)$, and then the TIP variant $(8.0 \pm$ $2.3 \%$ ). However, the percentage of segmental glomerular sclerosis (\%SS) was not significantly different among the variants. The total percentage of GS plus SS (\%GS + SS) differed significantly among the variants $(P=0.0105)$, with higher values for the COL $(41.9 \pm 6.1 \%)$, CEL $(42.2 \pm 5.5 \%)$, and NOS variants $(42.2 \pm 5.2 \%)$, followed by the $\mathrm{PH}$ variants $(32.8 \pm 4.0 \%)$ and the TIP variants $(25.1 \pm 2.7 \%)$. The IF/TA score also differed significantly among the variants $(P=0.0106)$; the CEL variant had the highest score $(2.10 \pm 0.27)$, followed by the NOS $(1.79 \pm 0.18)$, COL $(1.62 \pm 0.26)$, and PH variants $(1.54 \pm 0.18)$, with the TIP variant having the lowest score $(1.04 \pm 0.16)$.

Heterogeneity of segmental lesions in each histological variant

All glomeruli with segmental sclerosis were independently evaluated in terms of location and quality of the segmental lesions. The histological characteristics of segmental lesions in terms of location and quality factor are presented in Fig. 3. Segmental lesions were localized as TIP (Fig. 3a, b), PH (Fig. 3f) or NOS (Fig. 3c, d, e). Cellular segmental lesions were characterized by a proliferation of glomerular epithelial cells (Fig. 3a, c, d) and/or intracapillary occlusion with hypercellularity, including foam cells and infiltrated leukocytes (Fig. 3a, d). Fibrous lesions showed consolidation (sclerosis) of the glomerular tuft with increased extracellular matrix (Fig. 3b, e, f). Hyalinosis was observed in both cellular and fibrous lesions (Fig. 3f). The 210 glomerular segmental lesions consisted of 57 (27\%) cellular TIP, four (2\%) fibrous TIP, 42 (20\%) cellular NOS, $86(41 \%)$ fibrous NOS, and 21 (10\%) fibrous $\mathrm{PH}$ lesions (Fig. 4). The majority of segmental lesions in the TIP area were cellular (57/62, $93.4 \%$ ), and only a small percentage were fibrous $(5 / 62,8.1 \%)$. In contrast, all 21 segmental lesions in the $\mathrm{PH}$ area were fibrous. The segmental lesions in 


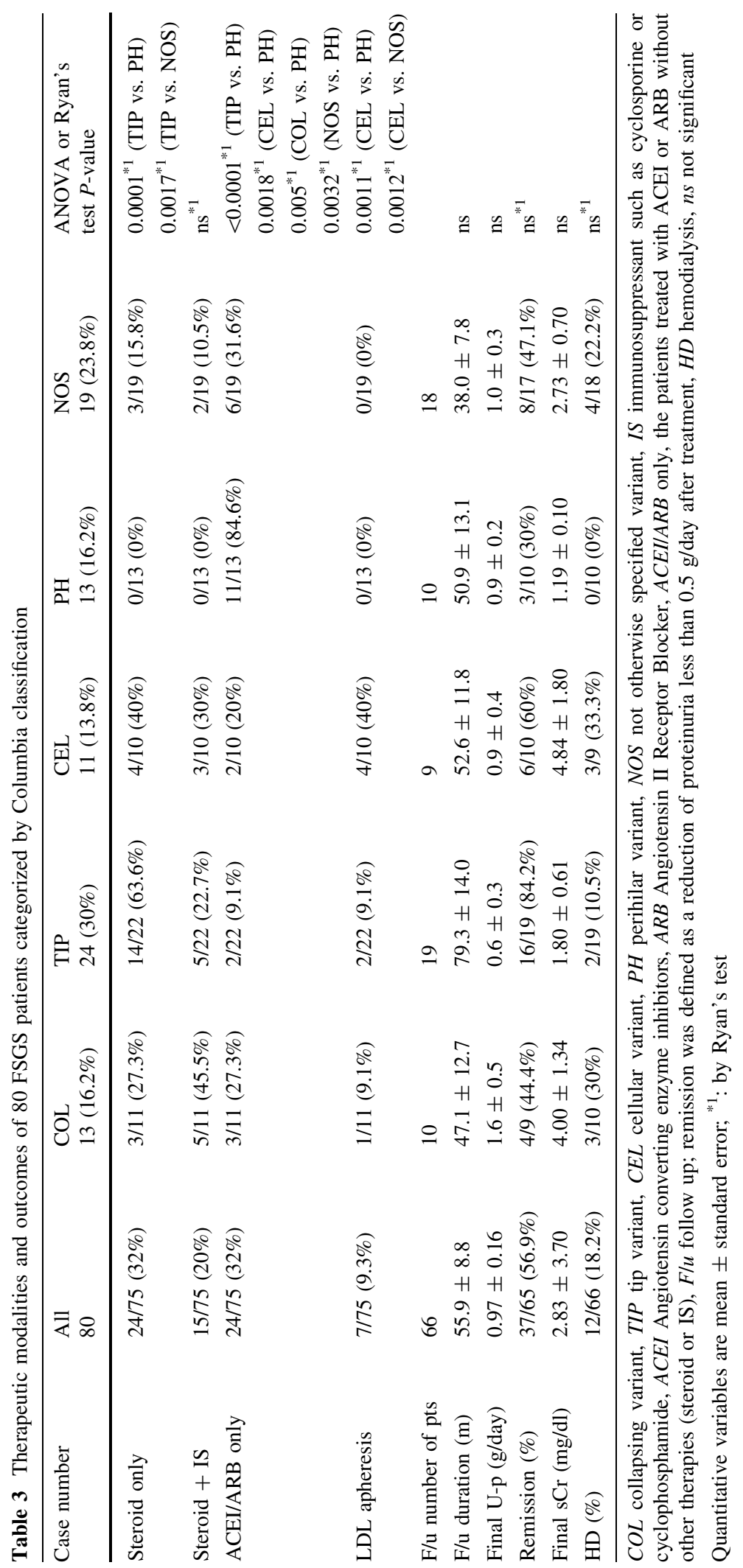


Table 4 Comparison of histological findings of 80 FSGS patients categorized by Columbia classification

\begin{tabular}{|c|c|c|c|c|c|c|c|c|}
\hline & All & $\mathrm{COL}$ & TIP & CEL & $\mathrm{PH}$ & NOS & $\begin{array}{l}\text { ANOVA } \\
P \text { value }\end{array}$ & $\begin{array}{l}\text { Multiple } \\
\text { comparison } \\
\text { (Sheffe) } P<0.05\end{array}$ \\
\hline$\% \mathrm{GS}$ & $16.6 \pm 1.9$ & $21.1 \pm 5.9$ & $8.0 \pm 2.3$ & $17.5 \pm 4.1$ & $16.8 \pm 2.9$ & $23.6 \pm 5.1$ & 0.0361 & ns \\
\hline$\% \mathrm{SS}$ & $18.9 \pm 1.3$ & $20.8 \pm 3.7$ & $17.1 \pm 2.0$ & $24.7 \pm 5.2$ & $16.0 \pm 13.0$ & $18.6 \pm 2.3$ & ns & \\
\hline$\% \mathrm{GS}+\mathrm{SS}$ & $35.3 \pm 2.1$ & $41.9 \pm 6.1$ & $25.1 \pm 2.7$ & $42.2 \pm 5.5$ & $32.8 \pm 4.0$ & $42.2 \pm 5.2$ & 0.0105 & ns \\
\hline IF/TA score $(0-3)$ & $1.53 \pm 0.10$ & $1.62 \pm 0.26$ & $1.04 \pm 0.16$ & $2.10 \pm 0.27$ & $1.54 \pm 0.18$ & $1.79 \pm 0.18$ & 0.0106 & CEL vs. TIP \\
\hline
\end{tabular}

$G l$ glomerular, $G S$ global sclerosis, $S S$ segmental sclerosis, IF/TA interstitial fibrosis and tubular atrophy

Quantitative variables are mean \pm standard error of mean

the NOS area were either cellular $(42 / 127,33.1 \%)$ or fibrous $(86 / 127,66.9 \%)$.

The location heterogeneity of segmental lesions in each histological variant is shown in Fig. 5. The different degrees of heterogeneity were probably associated with the diagnostic hierarchy of the Columbia classification, which validated the diagnostic priority in the order COL, TIP, CEL, PH, and NOS. In the COL variant, segmental lesions were located in the TIP $(12 / 51,23.5 \%)$, NOS (36/51, $70.6 \%)$, and $\mathrm{PH}$ areas $(3 / 51,5.9 \%)$. In the TIP variant, segmental lesions were located in the TIP $(49 / 75,65.3 \%)$ and NOS areas $(26 / 75,34.7 \%)$, but not in the PH area. Half of the patients with the TIP variant $(12 / 24,50 \%)$ had segmental lesions located in the NOS area, but the dominant location of the segmental lesions was in the TIP area in 21/24 $(87.5 \%)$ cases (Table 1$)$. All of the segmental lesions in CEL variants were located in the NOS area (28/28, $100 \%)$. The majority of the segmental lesions in $\mathrm{PH}$ variants were located in the PH area $(18 / 21,85.7 \%)$, with only three (14.3\%) in the NOS area. Among the 13 patients with the $\mathrm{PH}$ variant, three patients $(23.1 \%)$ had segmental lesions in the NOS area, but the $\mathrm{PH}$ area in all $\mathrm{PH}$ variants was the dominant location of the segmental lesions (Table 1). All NOS variant segmental lesions were located in the NOS area $(35 / 35,100 \%)$.

The quality heterogeneity of segmental lesions in each histological variant is shown in Fig. 6. Of the 51 COL variant segmental lesions, $32(62.7 \%)$ were cellular, and $19(37.3 \%)$ were fibrous. The cellular subtype was also predominant for the TIP (51/75, $68 \%)$ and CEL variants $(16 / 28,57.1 \%)$. In contrast, all segmental lesions of the NOS and PH variants were fibrous.
Overlap of histological findings in COL and CEL variants

Overlap of CEL lesions in the COL variants occasionally made it difficult to determine the variant. Because the Columbia classification defines the COL variant as "at least one glomerulus with segmental or global collapse with overlying podocyte hypertrophy and hyperplasia" [3], CEL lesions, defined as endocapillary hypercellularity occluding lumina with or without foam cells and karyorrhexis, can occasionally be admitted in the COL variant (Fig. 7). The coexistence of CEL lesions in the COL variants were observed in 9 of $13 \mathrm{COL}$ cases (Supplemental Table 1). Although proteinuria tended to be higher in the COL variants with CEL lesions, no significant difference was demonstrated.

Dominant location/quality factors of segmental lesions correlation with clinical findings

Figure 8 compares clinical parameters according to the dominant location and quality factor of each case. With respect to predominant lesion location, the mean interval between symptom onset and biopsy was significantly shortest for patients with lesions predominantly in the TIP location (4.8 \pm 1.1 months), followed by the NOS location ( $52.5 \pm 10.1$ months $)$, and the interval was longest for the $\mathrm{PH}$ location $(137.9 \pm 24.1$ months). With respect to predominant quality factor, the mean interval was shortest for patients with lesions of the cellular subtype (5.7 \pm 1.0 months), longer for the fibrocellular subtype (40.8 \pm 12.1 months), and longest for the fibrous subtype (105.0 \pm 15.4 months; Fig. 8a). Proteinuria at biopsy was highest for patients with the 

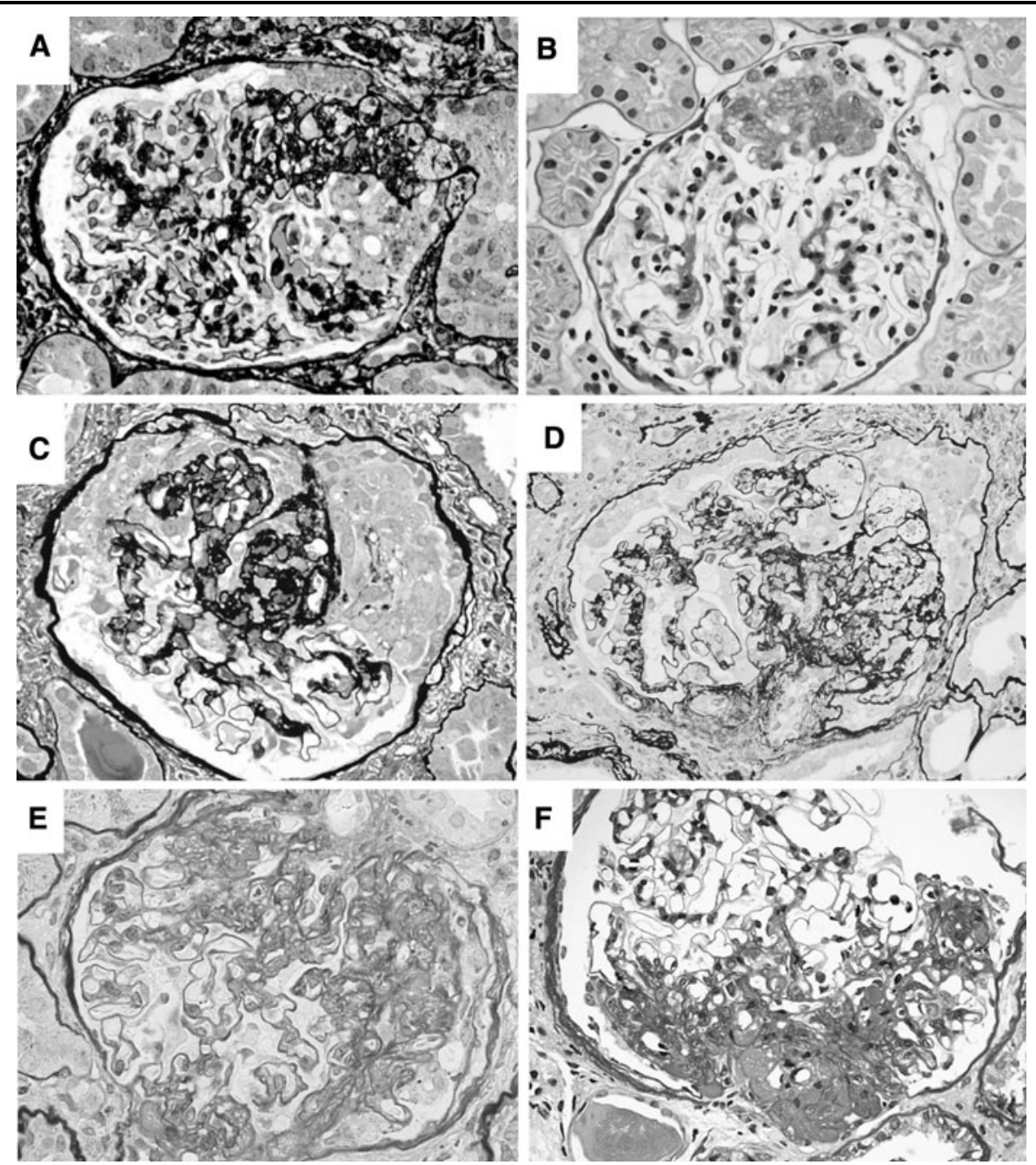

Fig. 3 Typical glomerular findings of segmental lesions regarding location and histological features. a TIP-located lesion with cellular histology (TIP-cellular). There is segmental accumulation of endocapillary foam cells involving the peripheral glomerular segment at the tubular pole. Podocytes overlying the segmental lesion are hypertrophic and hyperplastic. The histological variant of this glomerulus corresponds to TIP in the Columbia classification (silver methenamine stain, $\times 100$ ). b TIP-located lesion with fibrous histology (TIP-fibrous). The glomerular tufts are collapsed with segmental accumulation of extracellular matrix involving the peripheral glomerular segment at the tubular pole. Podocytes overlying the segmental lesion are slightly proliferative. The histological variant of this glomerulus also corresponds to TIP in the Columbia classification. As a TIPcellular lesion co-existed in the same specimen, the histological category of this case was deemed TIP-fibrocellular (F/C) (PAS stain, $\times 100)$. c Extensive segmental lesion in the NOS area with cellular histology (NOS-cellular). The glomerulus shows extensive collapse and obliteration of the glomerular tufts, accompanying prominent hypertrophy and hyperplasia of the overlying glomerular epithelial cells. This case is categorized as NOScellular and classified as a COL variant in the Columbia

classification (silver methenamine stain, $\times 100$ ). d Segmental lesion in the NOS area with active endocapillary cellular histology (NOS-cellular). Endocapillary accumulation of foam cells and leukocytes occluding the glomerular capillary lumina is regarded as an active histological feature of glomerular segmental lesions. This case is categorized as NOS-cellular and classified as a CEL variant in the Columbia classification. In addition to endocapillary proliferation, collapsing glomerular tufts may also be observed in some areas of the segmental lesion, making it difficult to differentiate between the COL and CEL variants (silver methenamine stain, $\times 100$ ). e NOS-located lesion with a predominantly fibrous appearance (NOS-fibrous). Extensive segmental sclerosis is observed involving the NOS area, but at neither the vascular nor tubular pole. No cellular proliferation is seen in the segmental lesion. The case is categorized as NOSfibrous and classified as NOS in the Columbia classification (PAS stain, $\times 100)$. f PH-located lesion showing segmental occlusion of the glomerular capillaries by fibrous matrix accumulation and hyalinosis around the glomerular hilum (PH-fibrous). Neither epithelial hyperplasia nor endothelial proliferation is seen in this glomerulus. This case is categorized as PH-fibrous and classified as the $\mathrm{PH}$ variant in the Columbia classification (PAS stain, $\times 100$ ) 


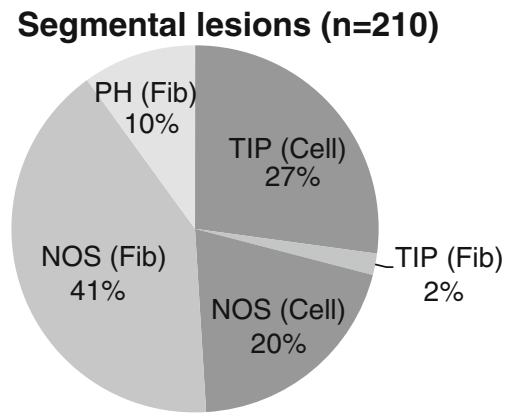

Fig. 4 Composition of location and quality factors in all segmental lesions $(n=210)$. The 210 glomerular segmental lesions consisted of 57 (27\%) cellular TIP, four (2\%) fibrous TIP, $42(20 \%)$ cellular NOS, $86(41 \%)$ fibrous NOS, and 21 (10\%) fibrous PH lesions. The majority of segmental lesions in the TIP area were cellular, whereas all 21 segmental lesions in the PH area were fibrous. The segmental lesions in the NOS area were mixed cellular $(33.1 \%)$ and fibrous $(66.9 \%)$

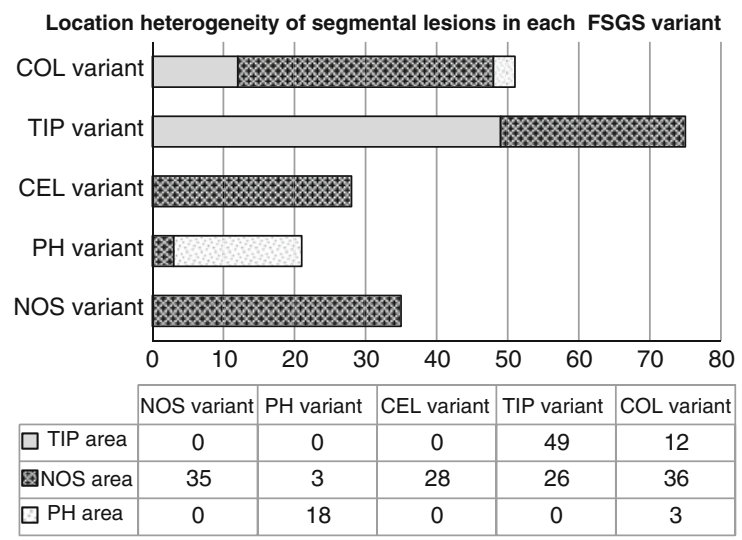

Number of segmental lesion in each location; TIP, NOS, or PH area in each FSGS variant.

Fig. 5 Location heterogeneity of segmental lesions in each histological variant. Heterogeneity in terms of location was present of the segmental lesions. In the COL variant, segmental lesions were located in the TIP $(12 / 51,23.5 \%)$, NOS $(36 / 51$, $70.6 \%)$, and $\mathrm{PH}$ areas $(3 / 51,5.9 \%)$. In the TIP variant, segmental lesions were located in the TIP $(49 / 75,65.3 \%)$ and NOS areas $(26 / 75,34.7 \%)$, but not in the PH area. All segmental lesions in the CEL variant were located in the NOS area $(28 / 28,100 \%)$. Most of the segmental lesions in the $\mathrm{PH}$ variant were located in the $\mathrm{PH}$ area $(18 / 21,85.7 \%)$, with three located in the NOS area $(3 / 21,14.3 \%)$. All segmental lesions in the NOS variant were located in the NOS area $(35 / 35,100 \%)$. Gray bars, TIP area; dashed bars, NOS area; dotted bars, PH area

predominantly TIP lesion location $(10.4 \pm 2.2 \mathrm{~g} /$ day $)$, followed by the NOS location $(4.6 \pm 1.0 \mathrm{~g} /$ day $)$, and the PH location $(2.1 \pm 0.4 \mathrm{~g} /$ day $)$. Proteinuria was highest in the cellular subtype $(11.8 \pm 1.9 \mathrm{~g} /$ day $)$,
Quality heterogeneity of segmental lesionsin each FSGS variant

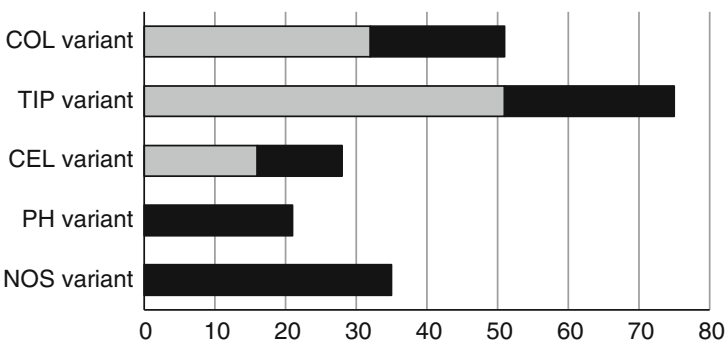

\begin{tabular}{|l|c|c|c|c|c|}
\cline { 2 - 6 } \multicolumn{1}{c|}{} & NOS variant & PH variant & CEL variant & TIP variant & COL variant \\
\hline$\square$ Cellular & 0 & 0 & 16 & 51 & 32 \\
\hline - Fibrous & 35 & 21 & 12 & 24 & 19 \\
\hline
\end{tabular}

Number of segmental lesions with cellular or fibrousg subtype in each FSGS variant.

Fig. 6 Quality heterogeneity of segmental lesions in each histological variant. The quality composition, cellular (white bars) and fibrous (black bars) segmental lesions, in each variant is shown. Among the 210 segmental lesions, 99/210 $(47.1 \%)$ were cellular and 111/210 (52.9\%) were fibrous. In the COL variant, 32/51 (62.7\%) were cellular and 19/51 (37.3\%) were fibrous. The cellular subtype was also predominant in the TIP $(51 / 75,68 \%)$ and CEL variants $(16 / 28,57.1 \%)$. In contrast, all segmental lesions were fibrous in the NOS and PH variants. Gray bars, cellular; black bars, fibrous

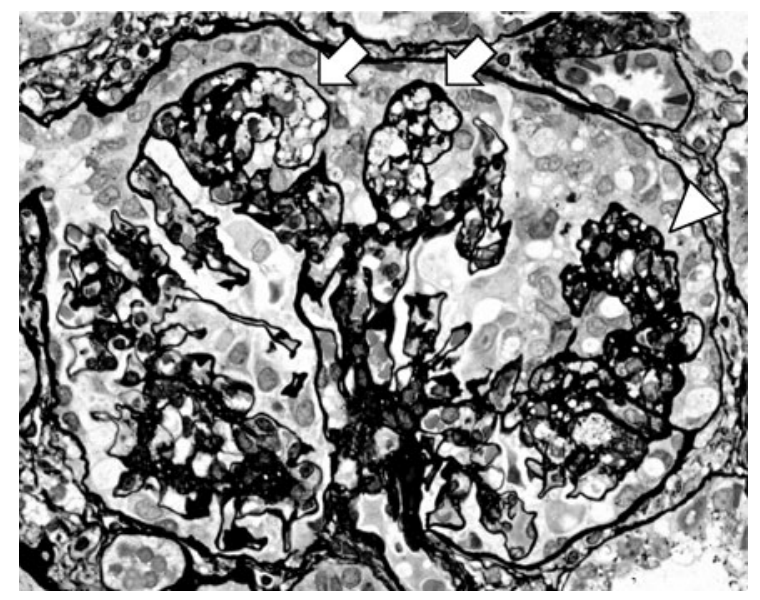

Fig. 7 Overlap of COL and CEL lesion histological findings. The glomerulus showed the coexistence of segmental tuft collapse (arrow head) and endocapillary hypercellularity with intracapillary foam cells (arrow). Overlap between the CEL lesion in the COL variant sometimes made it difficult to determine the variant. The CEL lesion was present in nine of 13 cases with COL variants in this study (silver methenamine stain, $\times 200$ )

followed by the fibrocellular $(3.4 \pm 1.0 \mathrm{~g} /$ day $)$, and fibrous subtypes $(1.9 \pm 0.3 \mathrm{~g} /$ day; Fig. $8 \mathrm{~b})$. The $\mathrm{sCr}$ level at final observation was highest for the 
predominantly NOS location $(4.0 \pm 0.8 \mathrm{mg} / \mathrm{dl})$ and was lower for the TIP location $(1.4 \pm 0.4 \mathrm{mg} / \mathrm{dl})$ and $\mathrm{PH}$ location $(1.2 \pm 0.1 \mathrm{mg} / \mathrm{dl})$, to a similar degree. However, the sCr level at the end of follow-up did not differ by the predominant quality factor: cellular, $3.2 \pm 0.9 \mathrm{mg} / \mathrm{dl}$; fibrocellular, $2.5 \pm 0.9 \mathrm{mg} / \mathrm{dl}$; and fibrous, $2.7 \pm 0.7 \mathrm{mg} / \mathrm{dl}$ (Fig. 8c). No significant difference was evident in the other clinical parameters, including the $\mathrm{sCr}$ level at biopsy and proteinuria at final evaluation.
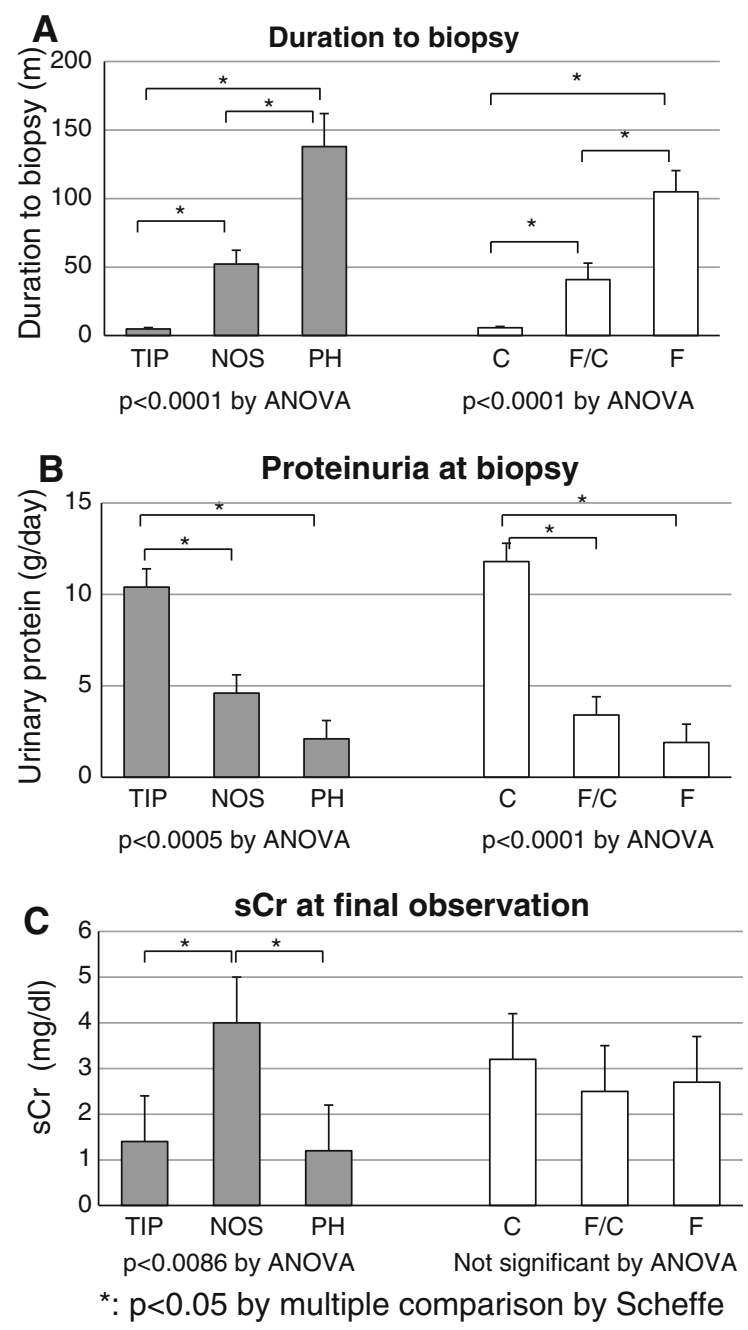

Fig. 8 Correlation between clinical parameters and dominant location/quality factors of segmental lesions. a Interval between onset and biopsy (months). b Urinary protein excretion at biopsy (g/day). c Serum creatinine ( $\mathrm{sCr}$ ) level at final observation ( $\mathrm{mg} / \mathrm{dl})$. Both the location and quality of lesions were significantly correlated with disease duration and proteinuria at biopsy. The outcome, as reflected by the $\mathrm{sCr}$ at the end of follow-up, was significantly correlated with location, but not with quality of lesions
Dominant location/quality factors of segmental lesions correlation with histological parameters

Globally sclerotic glomeruli represented a significantly lower percentage of total glomeruli in the TIP location $(4.2 \pm 1.1 \%)$ compared with the NOS $(22.6 \pm 3.0 \%)$ and $\mathrm{PH}$ locations $(19.0 \pm 3.1 \%)$, and were lower in the cellular subtype $(8.1 \pm 2.7 \%)$ than in the fibrocellular $(19.6 \pm 3.4 \%)$ and fibrous subtypes $(22.5 \pm 3.2 \%$; Fig. 9a). In contrast, the percentage of segmentally sclerotic glomeruli did not differ significantly among the location categories: TIP, $18.4 \pm 2.3 \%$; NOS, $20.3 \pm 2.0 \%$; and $\mathrm{PH}, 16.3 \pm 1.9 \%$. However, the percentage of segmentally sclerotic glomeruli was higher in the fibrocellular subtype $(25.7 \pm 2.6 \%)$ than in the cellular $(17.2 \pm 2.4 \%)$ and fibrous subtypes $(17.0 \pm 1.6 \%$; Fig. $9 \mathrm{~b})$. The IF/TA score for the TIP category $(0.91 \pm 0.17)$ was significantly lower than the score for the NOS category $(1.86 \pm 0.13)$, which was not significantly different from the score for the $\mathrm{PH}$ category $(1.53 \pm 0.17)$. Additionally, the IF/TA score was lower for the cellular subtype $(1.10 \pm 0.15)$ compared with the fibrocellular $(1.94 \pm 0.22)$ and fibrous subtypes ( $1.67 \pm 0.13$; Fig. 9c).

Clinical relevance of the location and quality heterogeneity in each variant

To examine the clinical relevance of the location and quality heterogeneity, we examined the COL, TIP, and PH variants with different location categories (Supplemental Tables 2-4) and the COL, CEL, and TIP variants with different quality categories (Supplemental Tables 5-7). The results revealed that the location and quality heterogeneity of segmental lesions have some influence on the clinical and histological manifestations in each variant of FSGS, but further study with a greater number of patients is required to show distinct statistical differences.

\section{Discussion}

The present study indicated that location heterogeneity of glomerular segmental lesions was observed in the COL and TIP variants, and indistinctly in the $\mathrm{PH}$ variant. The CEL and NOS variants showed a homogenous location, because of their diagnostic 

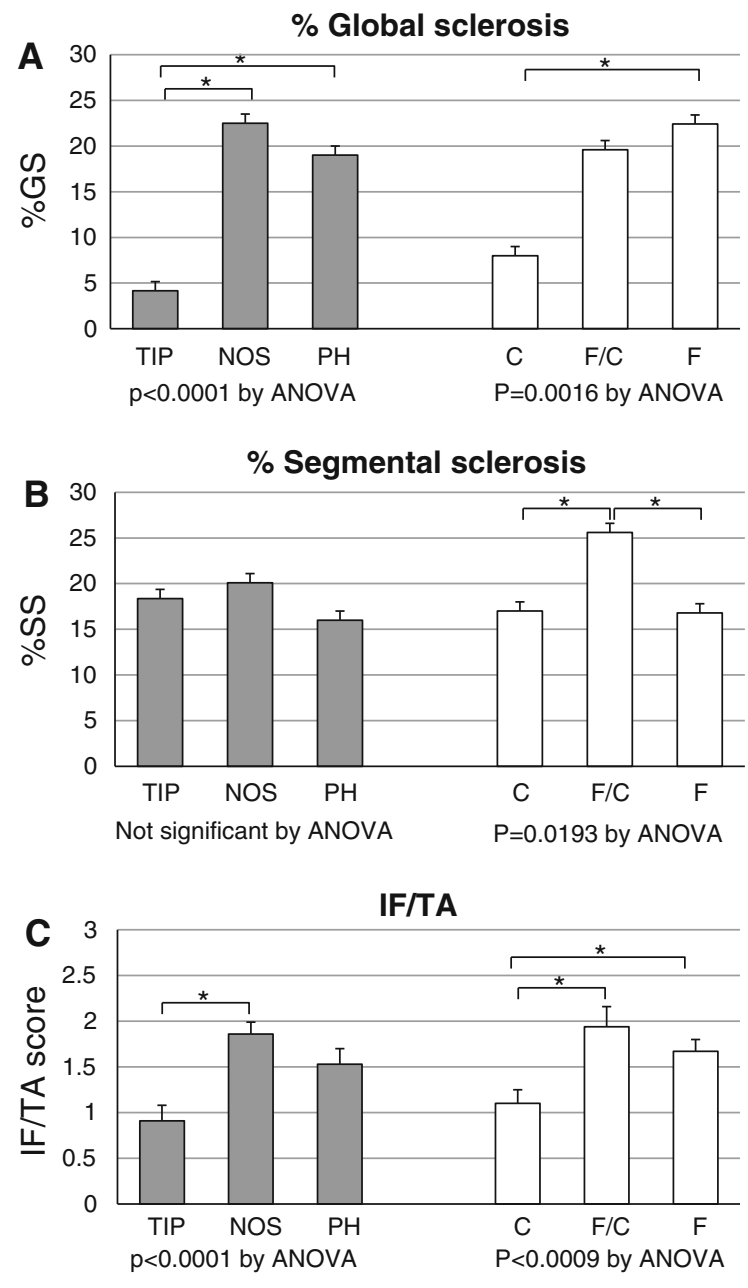

*: $p<0.05$ by multiple comparison by Scheffe

Fig. 9 Correlation between histological parameters and dominant location/quality factors of segmental lesions. a Globally sclerotic glomeruli as a percentage of the total number of glomeruli (\%GS). b Segmentally sclerotic glomeruli as a percentage of the total number of glomeruli (\%SS). c Score of interstitial fibrosis and tubular atrophy (IF/TA score). Both the location and quality of lesions were significantly correlated with \%GS and the IF/TA score. Quality was correlated only weakly and location not at all with \%SS, meaning that the percentage of segmentally sclerotic glomeruli was similar across location categories and quality subtypes

criteria. The clinical relevance of location heterogeneity was partly shown in the COL variant: a shorter duration to biopsy in the COL variants affecting the TIP area and a higher incidence of ESRD in the COL variants affecting the NOS area. The location factor for the segmental lesion may be important in assessing clinical and histological findings of the COL variants. The segmental COL lesion localized in the TIP or PH area may indicate less serious clinical manifestations than the typical COL variant, as reported previously [18-23]. In contrast, the clinical relevance of location heterogeneity was obscure in the TIP and PH variant, meaning that the detection of a single segmental lesion consistent with the TIP and $\mathrm{PH}$ variant is generally accepted as sufficient for determining variant classification.

Quality heterogeneity in terms of activity and chronicity of glomerular segmental lesions was also noticed in the COL, TIP, and CEL variants. The activity and chronicity of glomerular lesions are good indicators of the severity of various glomerular diseases [12-17]. Although histological activity and chronicity are controversial in FSGS, it is generally accepted that hypercellular lesions are frequently observed in patients with severe clinical manifestations, such as the COL or CEL variants of the Columbia classification. The present study also indicated that the cellular characteristics of segmental lesions were associated with a shorter delay to biopsy and severe proteinuria, and that the quality heterogeneity of segmental lesions reflected the different clinical and histological manifestations in the patients with COL and CEL variants.

Determining the dominant location and quality of glomerular segmental lesions may be useful for estimating clinical severity and for the histological classification of FSGS. The dominant location of lesions was significantly associated with several clinical and histological features, as originally suggested by D'Agati et al. [3]. Severe clinical manifestations and unfavorable outcomes were associated with the NOS location of segmental lesions, and a favorable outcome was associated with the TIP and $\mathrm{PH}$ locations, consistent with previous studies [5, 7, 17, 22]. The TIP location correlated well with severe proteinuria, shorter delay to biopsy, and favorable prognosis. The PH location was associated with the longest duration to biopsy, a lower level of proteinuria, and, similar to the TIP location, a favorable outcome. Similarly, the dominant quality factor (cellular, fibrocellular or fibrous) of lesions was significantly associated with the level of proteinuria at biopsy and the extent of glomerular sclerosis. The cellular subtype is associated with acute and severe clinical manifestation, and the fibrous subtype represents chronic and milder clinical presentation. Thus, the dominant quality as well as the dominant location 
of glomerular segmental lesions is a good indicator of clinical manifestations.

The distinction between the COL and CEL variants requires further investigation due to their overlapping histological findings and the apparent histological heterogeneity of glomerular segmental lesions. Endocapillary hypercellularity, a diagnostic criterion of the CEL variant, can also be encountered in COL variant cases. Overlapping histological findings in the COL and CEL variants sometimes cause difficulty in identifying the variant [1]. This situation may be associated with the result that no significant difference in the clinical and histological findings was seen between the COL and CEL variants. Further studies are needed to identify distinct differences between the COL and CEL variants as applied to cases of histologically overlapping COL and CEL findings. Furthermore, it remains to be determined which histological findings, for example, epithelial hyperplasia or endocapillary proliferation, are, in fact, associated with disease activity and progression in FSGS.

In conclusion, histological heterogeneity of glomerular segmental lesions exists in all variants of the Columbia classification, except NOS, and reflects the clinical diversity within each variant of FSGS. Determining the dominant location and quality subtype of glomerular segmental lesions may be useful for classifying variants and estimating clinical severity and outcome in patients with FSGS. Although the generally conserved fidelity of location and dominance of histological features in the TIP, PH, and NOS variants support the utility of the Columbia classification, the COL and CEL variants warrant further investigation due to their overlapping histological findings and apparent histological heterogeneity of glomerular segmental lesions.

Open Access This article is distributed under the terms of the Creative Commons Attribution Noncommercial License which permits any noncommercial use, distribution, and reproduction in any medium, provided the original author(s) and source are credited.

\section{References}

1. Schwartz MM, Evans J, Bain R et al (1999) Focal segmental glomerulosclerosis: prognostic implications of the cellular lesion. J Am Soc Nephrol 10:1900-1907
2. D'Agati VD (2008) The spectrum of focal segmental glomerulosclerosis: new insights. Curr Opin Nephrol Hypertens 17:271-281

3. D’Agati VD, Fogo AB, Bruijn JA et al (2004) Pathologic classification of focal segmental glomerulosclerosis: a working proposal. Am J Kidney Dis 43:368-382

4. Stokes MB, Markowitz GS, Lin J et al (2004) Glomerular tip lesion: a distinct entity within the minimal change disease/focal segmental glomerulosclerosis spectrum. Kidney Int 65:1690-1702

5. Thomas DB, Franceschini N, Hogan SL et al (2006) Clinical and pathologic characteristics of focal segmental glomerulosclerosis pathologic variants. Kidney Int 69: 920-926

6. Stokes MB, Valeri AM, Markowitz GS et al (2006) Cellular focal segmental glomerulosclerosis: clinical and pathologic features. Kidney Int 70:1783-1792

7. Howie AJ, Pankhurst T, Sarioglu S et al (2005) Evolution of nephrotic-associated focal segmental glomerulosclerosis and relation to the glomerular tip lesion. Kidney Int 67:987-1001

8. Silverstein DM, Craver R (2007) Presenting features and short-term outcome according to pathologic variant in childhood primary focal segmental glomerulosclerosis. Clin J Am Soc Nephrol 2:700-707

9. IJ DH, Farris AB, Goemaere N et al (2008) Fidelity and evolution of recurrent FSGS in renal allografts. J Am Soc Nephrol 19:2219-2224

10. Canaud G, Dion D, Zuber J et al (2009) Recurrence of nephrotic syndrome after transplantation in a mixed population of children and adults: course of glomerular lesions and value of the Columbia classification of histological variants of focal and segmental glomerulosclerosis (FSGS). Nephrol Dial Transplant 25:1321-1328

11. Schwartz MM, Korbet SM (1993) Primary focal segmental glomerulosclerosis: pathology, histological variants, and pathogenesis. Am J Kidney Dis 22:874-883

12. Weening JJ, D'Agati VD, Schwartz MM et al (2004) The classification of glomerulonephritis in systemic lupus erythematosus revisited. J Am Soc Nephrol 15:241-250

13. Shigematsu H (1997) Histological grading and staging of IgA nephropathy. Pathol Int 47:194-202

14. Taneda S, Honda K, Nitta K et al (1999) Clinicopathological study of IgA nephropathy in adults: the influence of onset age on clinical and renal histological findings and on the effect of steroid therapy. Clin Exp Nephrol 3:96-103

15. Honda K, Nitta K, Kobayashi H et al (2000) Clinical significance of histological grading and staging for predicting the effectiveness of steroid therapy in IgA nephropathy. Clin Exp Nephrol 4:241-250

16. Suzuki S, Joh K (2004) Applicability of steroid therapy in 275 adult patients with IgA nephropathy determined using a histological scoring system and degree of proteinuria. Clin Exp Nephrol 8:109-116

17. Cattran DC, Coppo R, Cook HT et al (2009) The Oxford classification of IgA nephropathy: rationale, clinicopathological correlations, and classification. Kidney Int 76: 534-545

18. Valeri A, Barisoni L, Appel GB et al (1996) Idiopathic collapsing focal segmental glomerulosclerosis: a clinicopathologic study. Kidney Int 50:1734-1746 
19. Detwiler RK, Falk RJ, Hogan SL et al (1994) Collapsing glomerulopathy: a clinically and pathologically distinct variant of focal segmental glomerulosclerosis. Kidney Int 45:1416-1424

20. Schwartz MM, Lewis EJ (1985) Focal segmental glomerular sclerosis: the cellular lesion. Kidney Int 28:968-974

21. Weiss MA, Daquioag E, Margolin EG et al (1986) Nephrotic syndrome, progressive irreversible renal failure, and glomerular "collapse": a new clinicopathologic entity? Am J Kidney Dis 7:20-28

22. Chun MJ, Korbet SM, Schwartz MM et al (2004) Focal segmental glomerulosclerosis in nephrotic adults: presentation, prognosis, and response to therapy of the histologic variants. J Am Soc Nephrol 15:2169-2177

23. Nair R (2006) Focal segmental glomerulosclerosis: cellular variant and beyond. Kidney Int 70:1676-1678 